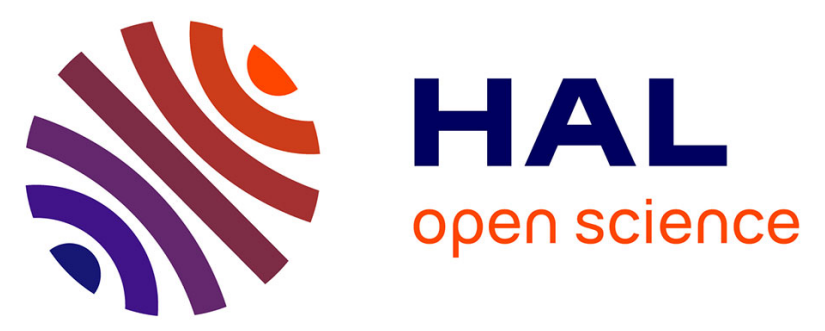

\title{
Exfoliation of Few-Layer Graphene in Volatile Solvents Using Aromatic Perylene Diimide Derivatives as Surfactants
}

Andrea Liscio, Konstantinos Kouroupis-Agalou, Alessandro Kovtun, Elias Gebremedhn, Mohamed El Garah, Wassima Rekab, Emanuele P Orgiu, Loris Giorgini, Paolo Samorì, David Beljonne, et al.

\section{To cite this version:}

Andrea Liscio, Konstantinos Kouroupis-Agalou, Alessandro Kovtun, Elias Gebremedhn, Mohamed El Garah, et al.. Exfoliation of Few-Layer Graphene in Volatile Solvents Using Aromatic Perylene Diimide Derivatives as Surfactants. ChemPlusChem, 2016, 82 (3), pp.358-367. 10.1002/cplu.201600503 . hal01872458

\section{HAL Id: hal-01872458 https://hal.science/hal-01872458}

Submitted on 20 Sep 2018

HAL is a multi-disciplinary open access archive for the deposit and dissemination of scientific research documents, whether they are published or not. The documents may come from teaching and research institutions in France or abroad, or from public or private research centers.
L'archive ouverte pluridisciplinaire HAL, est destinée au dépôt et à la diffusion de documents scientifiques de niveau recherche, publiés ou non, émanant des établissements d'enseignement et de recherche français ou étrangers, des laboratoires publics ou privés. 


\title{
Exfoliation of Few-Layer Graphene in Volatile Solvents Using Aromatic Perylene Diimide Derivatives as Surfactants
}

Andrea Liscio, ${ }^{*}$ [a] Konstantinos Kouroupis-Agalou,[a] Alessandro Kovtun,[a] Elias Gebremedhn,[d] Mohamed El Garah,[b] Wassima Rekab,[b] Emanuele Orgiu,[b] Loris Giorgini,[c] Paolo Samorì,[b] David Beljonne,[d] and Vincenzo Palermo*[a]

[a] Dr. A. Liscio, Dr. K. Kouroupis-Agalou, A. Kovtun, Dr. V. Palermo; ISOF, CNR, via Gobetti 101, 40129 Bologna (Italy)

E-mail : andrea.liscio@isof.cnr.it, vincenzo.palermo@isof.cnr.it

[b] Dr. M. El Garah, W. Rekab, Dr. E. Orgiu, Prof. P. Samorì; ISIS \& icFRC, Universit8 de Strasbourg\&CNRS, 8 all8e Gaspard Monge, 67000 Strasbourg (France)

[c] Prof. L. Giorgini; Dipartimento di Chimica Industriale "Toso Montanari" Universit/ di Bologna, viale Risorgimento 4, 40100 Bologna (Italy)

[d] E. Gebremedhn, Prof. D. Beljonne; University of Mons, Place du Parc 20, 7000 Mons (Belgium)

\begin{abstract}
Commercial, aromatic perylene diimide (PDI) dyes were used to exfoliate few-layer graphene nanosheets in low-boiling or- ganic solvents such as chloroform and tetrahydrofuran. Importantly, in such solvents, graphene cannot be exfoliated in the absence of the aromatic perylene diimide (PDI) dyes. The PDIs are physisorbed onto the basal plane of the nanosheet surface, which stabilized them in solution; the aromatic core lies flat on graphene and the PDI side groups influenced the physisorption strength and molecular packing. Upon varying just a single atom in the chemical structure of the side groups, significantly different exfoliation efficiencies were observed. The graphene-PDI interaction was studied at the nanoscale by scanning tunneling microscopy and molecular dynamics, at the microscale by atomic force and electron microscopy, and at the macroscale by optical spectroscopy. Thanks to the high volatility of the chosen solvent, the nanosheets can be embedded in standard polymer composites through a simple solvent-induced swelling procedure.
\end{abstract}

\section{INTRODUCTION}

Graphene is among the most promising new materials, togeth- er with graphyne[1] and pentagraphe,[2] and is attracting great interest because of its outstanding electrical, mechanical, thermal, and optical properties. With such unique characteristics, different kinds of graphene-based materials (GRMs) such as single- and few-layer graphene have been produced for applications in diverse technological fields including (opto)electronics, photonics, and energy generation and storage. In view of their high surface-to-volume ratio, GRMs are particularly appealing as nanofillers for polymer nanocomposites, especially for structural applications in the aerospace, automotive, and sporting tool industries, and so on. The number of final products on the market containing graphene-based composites is increasing continuously, including, for example, tennis rackets,[3] bicycles, and skis. 
One main advantage of graphene over other nanofillers such as carbon nanotubes is that its properties can be tuned by the method used for its production, which can involve top- down or bottom-up approaches.[4]

Dispersions of GRM can be obtained at low cost on the kilogram scale by liquid-phase exfoliation (LPE) of graphite, as pioneered by Coleman and co-workers.[5] This method is the most promising for industrial applications, for example, to produce graphene as a nanoadditive in polymer composites,[6] or ultra-light foams or 2D composites for electronics or energy storage.[7]

The principle of LPE is simple: graphite powder is inserted in a given solvent, and this liquid is exposed to ultrasound or high-speed mixing for a specific time. The effect of cavitation bubbles or mixing will create shear forces able to detach graphene sheets from the graphite powder.[8] In LPE, it is possible to enhance the exfoliation yield by exploiting the capacity of the molecules present in solution to adsorb on the graphene sheets, thereby preventing its restacking. Hitherto, a wide range of different molecules has been used in the LPE process. These can generally be divided in two main classes:

A) Organic solvents, with a surface tension of $40-50 \mathrm{~mJ} \mathrm{~m}^{-2}$, which matches the surface energy of graphite.[9] The most suitable media for liquid formulations used presently are highboiling solvents such as $\mathrm{N}, \mathrm{N}$-dimethylformamide (DMF) and $\mathrm{N}$-methyl-2-pyrrolidone (NMP). A major disadvantage of using such solvents is their difficult removal.

B) Amphiphilic surfactants consisting of a hydrophobic tail, which has a high propensity to physisorb on the basal plane of graphene,[10] and a hydrophilic head-group, which favors solubility in water. Besides the use of "conventional" aliphatic surfactants (i.e. , soaps), stable dispersions of graphene in water have also been obtained using small poly- aromatic surfactants,[11] that is, molecules featuring a hydro- phobic, aromatic core, able to interact with graphene through $p-p$ stacking, and hydrophilic side groups.[12] A major advantage of this approach is that one can successfully use as surfactants some low-cost dyes that are already well established for the large-scale compounding of polymers, such as industrial additives and colorant dyes, which are able to exfoliate and stabilize graphene in water.[13] Notably, these molecules demonstrate effective exfoliation not only of graphene but also a wide range of different $2 \mathrm{D}$ materials (2 DMs) such as boron nitride, tungsten disulfide, and molybdenum sulfides, selenides, and tellurides.[14]

Albeit the most common and least polluting commonly used solvent, water is not the ideal solvent for every application. Although its boiling point is much lower than that of DMF (b.p. $=153^{\circ} \mathrm{C}$ ) or NMP (b.p. $=202{ }^{\circ} \mathrm{C}$ ), it is difficult to remove it quantitatively. Trace leftovers are detrimental, for example, acting as charge traps and oxidative defects in materials for electronics.[15] Moreover, in composites, water-based dispersions are not miscible with the vast majority of common thermoplastic polymers such as polypropylene (PP), polystyrene (PS), polyethylene terephthalate (PET), polyvinyl chloride (PVC), and so on.

Alternatives to NMP and DMF have been demonstrated to process graphene with semiconducting polymers, for example, the exfoliation of graphene with dichlorobenzene (DCB), but this solvent is toxic and has a high boiling point (189 8C).[16]

For an effective, technologically competitive application of 2DMs as additives in (nano)composites for electronics or structural applications it is preferable to solubilize graphene in low- boiling volatile solvents, such as those commonly used in organic chemistry laboratories, for example, chloroform 
(CHCl3) or tetrahydrofuran (THF). However, the use of amphiphilic surfactants is not suitable for solubilizing graphene in organic sol- vents, the polarities of which are typically low.

Therefore, we undertook a different strategy relying on the use of molecules featuring an extended polyaromatic core, which can interact through $p-p$ stacking with graphene. Such molecules are decorated with flexible side groups possessing a low but tunable polarity that makes them soluble in a wide range of organic solvents.

Perylene di-imide (PDI, Figure 1 a) has been chosen as the aromatic core because it represents a standard building block exploited in the fabrication of semiconducting materials for applications in electronics and photovoltaics.[17] It is also widely employed as a dye for textile applications and industrial paints as well as in protein tagging.[18]

As side groups, we have chosen relatively flexible phenylethyl moieties, which are able to render the perylene core soluble in many organic solvents. Owing to the complex interplay of supramolecular interactions of the PDI dyes with the solvent and with one another,[19] the self-assembly and solubility of the PDI dyes can already be tuned significantly simply by changing the most external atom of the side group.

Therefore, we performed a comparative and quantitative study on the interaction of three different PDI dyes with graphene $(G)$ sheets in solution. The only difference between the PDI dyes tested was the terminal atom in the side groups. In particular, we used a perylene core with side ethyl-phenyl group exposing in the para position a hydrogen (PDI@H), fluorine (PDI-F), or chlorine atom (PDI$\mathrm{Cl})$. This single atom change in the molecular structure is sufficient to modify the polarity of the flexible side chains and solubility behavior, but the optoelectronic properties of the perylene core remain unchanged. In general, it is well known that the supramolecular self-assembly, that is, the way the molecules interact if physisorbed on a flat solid surface, depends on substituents, as they rule intermolecular interactions through weak forces.[20] Scanning tunneling microscopy (STM) imaging provides unambiguous evidence that single atom substitutions lead to a dramatic change in the supramolecular packing of PDI dyes on highly oriented pyrolytic graphite (HOPG) (Figure 1 b,c,d).

We have demonstrated that all three PDI dyes tested are able to stabilize few-layer graphene flakes in dispersion to give graphene-organic hybrids (GOHs). Their different supramolecular behavior influences significantly their interaction with gra- phene, yielding GOHs with good stability in different solvents, and good processability as polymer additives.

These GOHs were characterized extensively at the nanoscale using STM, AFM, and SEM. The details of the PDI-graphene interaction at the atomic scale were also modelled by force-field calculations, to estimate the contributions of the different parts of the PDI dyes to the interactions, and the effects of the single atom difference between the PDI dyes.

In general, exfoliation with organic molecules never provides a high yield of few-layer graphene (FLG) (i.e., , 5 monoatomic sheets), unlike exfoliation proceeding by chemical or electro- chemical oxidation.[8] This approach has been generalized successfully to produce other 2D materials such as boron nitride (BN) and transition metal dichalcogenides (TMDs).[21] Purification of the monolayers is achieved by centrifugation steps[22] or by chromatography[23] for applications such as transparent conductors, for which monolayers are required.

Herein, we instead use these materials, with no purification by centrifugation, as additives in polymer composites or in electronics to enhance the electrical properties of the polymer in a thin film or in the bulk matrix. In such applications, low- cost and fast, facile processability are key factors 
; the coating of organic dyes enhances the interaction with the polymeric matrix, and recent results indicate that FLG could even provide better structural reinforcement than pure monolayers for appli- cations in polymer composites.[24]

The stability of such GOHs in volatile solvents allows their more facile use for typical target applications of graphene- based materials. To demonstrate this, we used the GOHs produced in this way as electrically conductive additives to embed them into standard polymer films.

\section{RESULTS AND DISCUSSION}

Figure 1a displays the chemical structure of the commercial PDI dyes under study. Previous reports have demonstrated clearly that a key step in graphite exfoliation in the presence of any kind of polyaromatic surfactant is the adsorption of the aromatic dyes on the graphene surfaces to stabilize them in liquid media and thereby hinder their re-aggregation.[25] Therefore, this important step was studied at the molecular, microscopic, and macroscopic scales.

\section{Study of G-PDI interactions at single-molecule level}

The adsorption of single PDI dyes on a graphene sheet was first investigated by using force-field simulations (see Supporting Information for details). Each of the three PDI dyes was positioned on the surface of graphene in a box of solvent molecules modelled explicitly, and simulated for a total of $10^{5}$ ns. $\mathrm{CHCl}_{3}$ was chosen as a model solvent.

The inset of Figure 2 shows some snapshots taken from simulations of three PDIs on graphene, and the statistics showing the average distance between the phenyl rings and the graphene for the different PDI dyes investigated. Figure S1 (Sup- porting Information) reports the calculated interaction of the PDI dyes with the solvent and with the graphene surface.

As expected, all the molecules physisorb preferentially with the PDI core flat on the substrate, whereas the phenyl side groups adopt a tilted arrangement. Significantly, the change in the single atom in the side groups leads to significantly different self-assembly behaviors. Although the strongest molecule- graphene interaction involves the aromatic core of the molecule, the $H, F$, and $\mathrm{Cl}$ atoms in the side-chains alter their ad-sorption energies on graphene.

The phenyl rings of the central aromatic core interact strongly with graphene, and reside close to the surface by packing parallel to it, with an average distance of $0.38 \mathrm{~nm}$ be- tween graphene and the molecule center of mass (peak P1 in Figure 2). In the case of hydrogen (PDI-H) and fluorine (PDI-F) terminations, the phenyl rings were found to rotate perpendicular to the surface, thereby increasing the graphene-molecule distance to $0.48 \mathrm{~nm}$ (peak P2); in particular, in PDI-F the phenyl ring could fold onto the PDI core (peak P3). A more de-tailed description of the modelling results is available in the Supporting Information.

Chlorine-terminated PDIs showed a much stronger interaction with graphene by adopting a flat conformation on the surface. The calculated interaction energy between the side groups and graphene (Table S1, Supporting Information) amounts to $20.04 \pm 0.95 \mathrm{kcal} \mathrm{mol}^{-1}$ for PDI-Cl, which is significantly larger than of the values for PDI-H and PDI-F $\left(15.86 \pm 1.14\right.$ and $16.40 \pm 0.91 \mathrm{kcal} \mathrm{mol}^{-1}$, respectively). 
The differences between these values are comparable to the adsorption energy of a single $\mathrm{C}$ atom of the adsorbate in contact with graphene,[26] suggesting the importance of single atom substitution in the 2D supramolecular arrangement of small aromatic dyes such as PDIs.

\section{Study of G-PDI interactions on nanometer scale}

The computational study of single molecules adsorbed on graphene can be useful to gain an insight into the molecule-surface interactions, but it cannot provide information on the collective behavior of these self-assembling PDIs. Therefore, we studied the adsorption of the three different PDIs on graphite substrates by means of STM at the solid/liquid interface. STM imaging reveals that all three PDIs are able to form tightly packed, 2D crystals on graphite (Figure 1 b,c,d and Table S1, Supporting Information). Whereas PDI-H and PDI-F exhibit comparable unit cell areas $(1.96 \pm 0.16$ and $2.10 \pm 0.16$ $\mathrm{nm}^{2}$ molecule $\mathrm{e}^{-1}$, respectively), PDI-Cl displays significantly denser packing, with a unit cell area corresponding to $1.68 \pm 0.13 \mathrm{~nm} 2$ molecule $^{-1}$ (i.e. , surface density $=0.60 \pm 0.05$ molecule $\mathrm{nm}^{-2}$ ). This last value is in agreement with the surface density calculated by Kozlov et al. ,[27] amounting 0.595 mole-culenm@2 for a high-coverage brick-wall arrangement of the perylene diimide molecule.

This denser packing was not caused by steric hindrance, given that the chlorinated groups are larger and more bulky than the protonated and fluorinated ones, nor can it be explained by the higher polarity of the chlorophenylethyl moiety (2.56 Debye), because PDI-F and PDI@H show a similar packing on graphene even though the former has highly polar side groups (2.23 Debye) whereas the latter does not ( 0.42 Debye). The different packing of PDI-Cl can instead be explained by a stronger interaction of the chlorinated side groups with graphene, in good agreement with the force-field calculations, which encourages more PDI to be adsorbed on the surface regardless of the increased steric hindrance. The density of molecules that can self-assemble to form a monolayer on a surface depends on the interplay between molecule- molecule and molecule-surface interactions, as we described previously in Ref.[19a] . Every time a new molecule adsorbs on graphene, the energy of the system decreases owing to favorable graphene-PDI interactions $\left(=20.04 \mathrm{kcal} \mathrm{mol}^{-1}\right.$ for PDI-Cl, as mentioned above); however, there will also be an increase in energy owing to the repulsive interaction of adsorbed molecules (caused by steric hindrance).

The number of adsorbed molecules will increase until these two factors reach an equilibrium. For $\mathrm{PDI}-\mathrm{Cl}$, the interaction with graphene is stronger than for the other PDIs, so equilibrium will be reached at a higher surface density.

Overall, the STM data show that all three PDI are able to interact strongly with graphite, albeit with different strengths, forming ordered monolayers on it. Unfortunately, STM at the solid/liquid interface needs to be performed by using high- boiling solvents, so it cannot be applied to explore the interaction of dyes with graphite or graphene in low-boiling solvents. To overcome this issue, we studied interaction of the three PDIs with graphite at the macroscopic level, in chloroform and THF, using a "dye capture" technique that has already been used successfully for water solutions.[13]

\section{Study of G-PDI interactions on macroscopic scale}

In this simple yet effective experiment, we compared the relative adsorption from solution of the different dyes onto the graphite surface. Increasing amounts of graphite powder with known surface area were incubated in solutions of the different PDIs. The amount of PDI adsorbed on the surface of the graphite powder could then be measured very precisely by monitoring the decrease in optical 
absorption of the solution. We underline that no sonication was applied to the system and no exfoliation process took place, so the experiment is a plain measure of the relative affinity of the different PDIs for graphite.[25]

Our approach is similar to that used in the measurement of Brunauer-Emmett-Teller (BET) specific surface areas of materials, monitoring the change in pressure of a gas owing to ad-sorption on the surface, or in water purification tests using organic dyes such as methylene blue.[28]

Figure3 shows the average amount of PDI adsorbed on graphite (NADS) versus the inverse of the available area of graphite $\left(1 / S_{A}\right)$. For low $S_{A}$ values (right part of the graph), $N_{A D S}$ is low because there is not enough graphite surface available for the molecules to adsorb.

Upon addition of more graphite, $S_{A}$ increases (i.e. $1 / S_{A}$ decreases), eventually reaching a threshold point, which is clearly visible as a sudden change in the slope in all the graphs. Above this threshold (left of each graph), nearly all molecules are adsorbed, so a plateau is visible in the graphs.

Some deviations are observed depending on the molecule and solvent used. In particular, PDI-Cl shows more irregular ad- sorption behavior, probably caused by the denser packing and strong interaction with graphite, as shown by the modelling results.

The threshold point observed in such "dye capture" experiments corresponds to a fully covered (i.e. , saturated) graphite surface on which no more molecules can be adsorbed. The observed threshold corresponds fairly well with the molecular footprint estimated on a nanometer scale by STM (blue arrows in Figure 3), suggesting that PDI molecules do not stack, but form a uniform single layer on FLG.

\section{Graphite exfoliation with different PDIs}

After quantification of the interaction of the PDIs with bulk graphite, we studied the exfoliation mechanism itself. The three PDIs were dissolved in a wide range of organic solvents of different polarities and increasing surface tensions : ethanol (EtOH), isopropyl alcohol (IPA), tetrahydrofuran (THF), chloroform ( $\mathrm{CHCl} 3)$, toluene, dichlorobenzene (DCB), dimethylformamide (DMF), and $\mathrm{N}$ Methyl-2-pyrrolidone (NMP).

The PDIs showed different solubilities in the different solvents, as also visible to the naked eye (Figure $4 \mathrm{a}, \mathrm{b}, \mathrm{c}$ ). As expected, each dye showed significantly different absorption spectra in the different solvents, owing to their different self-assembly properties and the influence of the solvent (Figure S2, Supporting Information).

These changes in optoelectronic properties of PDIs in sol tion have been studied extensively in previous work, and will not be discussed here ; for more details see, for example, reference [29] and references therein. Graphite powder was then added, and the solutions were sonicated exhaustively, following well-established standards.[9]

After sonication, the dispersions were centrifuged to remove larger aggregates, and the supernatant was taken for further analysis, with no additional purification steps. Figure $4 \mathrm{~d}$,e,f shows photographs of the resulting dispersions. Note that in some cases (e.g. , in IPA) the only visible effect of the treatment was the removal of the molecules leading to clean solvent purification, similar to the "dye capture" experiments, performed without sonication, described in the previous section. In most cases, however, dispersions of black graphitic material and PDI (named G-PDI) were obtained after centrifugation. The dispersions were stable after several months. The amount of dispersed 
material was measured precisely through optical ab- sorption spectroscopy (OS) of the diluted dispersions.

Optical absorption measurements of these dispersions showed spectra similar to those observed previously in graphene-pyrene composites, with well-defined adsorption peaks attributed to the PDI aromatic core, and a slowly decaying ab- sorption component typical of graphene (Figure S3, Supporting Information). The different optical properties of graphene and organic molecules in GPDI allow the estimation of their ratio by measuring the optical absorption at different wavelengths. The concentration of exfoliated graphene was estimated by measuring the absorbance at $650 \mathrm{~nm}$ (at which only the graphene absorbs), using the extinction coefficient reported in Ref. [9] . The concentrations of PDIs were obtained by measuring the absorption at the PDI strongest absorption wavelength ( $527 \mathrm{~nm}$ in $\mathrm{CHCl} 3 ; 521 \mathrm{~nm}$ in THF) after subtracting the contribution of graphene at that wavelength as found by deconvolution procedures. The molar extinction coefficient of each PDI was measured experimentally using solutions of known concentrations.

Figure 5a shows a plot quantifying the amount of dispersed graphitic material, for different solvents, for each PDI added to the solvent. The results obtained in pure solvent, without PDI, are also shown (white bars). From the plot, we can distinguish three different groups :

1) High-boiling solvents with a high surface tension of over $35 \mathrm{~mJ} \mathrm{~m}^{-2}$ (DCB, DMF, NMP, located on the right part of Figure $5 \mathrm{a}$ ), which yield high concentrations of dispersed graphitic materials regardless of the presence or absence of PDI. This result could be expected, because solvents such as DMF and NMP with a surface tension of $40-50 \mathrm{~mJ} \mathrm{~m}^{-2}$ are the best match for the graphite surface energy.[9] DCB has also been used successfully to exfoliate graphene, as shown, for example, in Ref. [16] . In these solvents, the addition of the PDIs is not significant ; it can give either an in- crease or a decrease in the amount of dispersed material.

2) Low-boiling, highly polar solvents (EtOH and IPA, located on the left part of Figure 5 a) with a surface tension below $25 \mathrm{~mJ} \mathrm{~m}^{-2}$, in which graphene is poorly soluble, regardless of the presence of PDI. Even the solubility of the PDIs was low in these solvents.

3) The middle part of Figure 5 a shows instead low-boiling sol- vents, with an average surface tension of $25-30 \mathrm{~mJ} \mathrm{~m}^{-2}\left(\mathrm{THF}, \mathrm{CHCl}_{3}\right.$ ). Sonication of graphite in the latter solvents yields typically very low concentrations of dispersed graphene derivatives. In these solvents, the addition of PDI increases greatly the amount of dispersed graphitic material (red arrows in Figure 5 a)

Notably, the efficiency of PDIs as dispersing agents does not seem to be related to the strength of their interaction with graphene ; $\mathrm{PDI}-\mathrm{Cl}$, the molecule with the highest adsorption energy and packing density on graphene, did not give the best results.

The relative improvement in exfoliation and dispersion be- cause of each PDI as a function of the solvent surface tension is plotted in Figure $5 \mathrm{~b}$. In most cases, a significant improvement was observed, with a maximum increase of about $1300 \%$ for PDI-F in THF. The addition of these molecules allows the exfoliation of graphite in solvents with surface tensions between 25 and $30 \mathrm{~mJ}$ $\mathrm{m}^{-2}$, which are significantly lower than those of DMF or NMP, typically used for graphene exfoliation (i.e. , 40-50 $\mathrm{mJ} \mathrm{m}^{-2}$ ).[9]

Tables 1 and 2 show the estimated amounts of dispersed FLG and PDI obtained, and the ratio R between the two (ex-pressed in graphene carbon atoms present in dispersion/PDI molecules present in dispersion, to allow comparison with STM data). We can see that, for poor surfactants 
(e.g. PDI-H in THF), the material dispersed in the solvent is mostly PDI (R\& 1). Conversely, for systems in which the PDI seems to have a positive effect (e.g. , in $\mathrm{CHCl}_{3}$ ), $\mathrm{R}$ is greater than 50 . Notably, good results were also observed for PDI-H, which is itself not very soluble in chloroform. This suggests the presence of a synergic effect between PDI@H and FLG, with each one stabilizing the other in solution.

The actual coverage of the PDI on the exfoliated flakes cannot be quantified, because exfoliation always yield a poly- disperse material featuring a range of several thicknesses, with different fractions of monolayers, bilayers, and thicker layers. $[8,25]$ The presence of graphene multilayers increases the number of carbon atoms in the dispersion. Consequently, the final $R$ values are systematically larger than those calculated from STM (Figure 1) assuming a perfect PDI layer adsorbed on completely exfoliated graphene sheets.

In the case of THF, the highest improvement in exfoliation, as compared to blank solvent, is achieved by using PDI-F and PDI-Cl (blue squares and red triangles in Figure $5 \mathrm{~b}$ ). However, a corresponding low $R$ value $(R=22)$ is measured, owing to the presence of large amounts of both flakes and PDI molecules that remain in the dispersion, probably as thick layers on the surface of the flakes. The presence of thick aggregates is also demonstrated by the onset of a new absorption band at $565 \mathrm{~nm}$ (white arrow in Figure S3, Supporting Information), in agreement with results reported in previous work.[30]

Exfoliation with PDI in $\mathrm{CHCl} 3$ gave lower concentrations of exfoliated material, but also higher $\mathrm{R}$ values. Thus, THF solvent is preferable if high absolute concentrations are needed, whereas $\mathrm{CHCl} 3$ is better if, instead, high nanosheet/PDI ratios are required.

The flakes obtained by exfoliation were characterized by AFM after spin coating on SiOx (Figure 6 a). Exfoliation with PDI in both solvents gave a uniform distribution of platelets with a broad thickness distribution ranging from $5 \mathrm{~nm}$ up to around $150 \mathrm{~nm}$, and an average lateral size of $150: 80 \mathrm{~nm}$. The minimal thickness obtained is much larger than that of monolayer graphene, indicating that the material obtained is a mixture of few-layer graphene and thicker sheets. Unfortunately, it is not possible to make more quantitative assessment of the thick- ness distributions of the sheets because of the unknown number of PDI molecules adsorbed on each sheet.

A similar result was obtained for graphene exfoliated in water using pyrenes; $; 14]$ in that case, Raman analysis confirmed that $50-60 \%$ of the sheets were two to seven layers thick, with a $10-20 \%$ fraction of monolayers, the rest having over seven layers. Unfortunately, the presence of intense PDI Raman features prevents any direct analysis by Raman spectroscopy.

Owing to the high thickness measured by AFM and the lack of Raman signal, the material obtained cannot be defined graphene, but rather, few-layer graphene (FLG).

\section{Processing of G-PDI in polymer composites}

Finally, the obtained materials were used directly to produce polymer composites for electrically conductive (antistatic or dissipative) applications.

The processing of graphene and GRMs with polymers is a major issue hindering widespread commercialization of graphene-based products. Processing of graphene composites by extrusion and solvent casting, molding, and so on requires careful tuning of the processing conditions and the nanosheet structure. Incorrect processing can lead to aggregation of the nanosheets, yielding 
mechanical defects and poor electrical percolation in the final composite, and modifying the rheology of the matrix and the final performance of the material.

To this end, we have used a technique recently developed by Coleman and co-workers for rubber, which is based on swelling a preformed polymer into a solution of FLG in NMP,[31] allowing the graphene sheets to penetrate into the rubber to obtain graphene-rubber composites.

By using, in our case, FLG dispersed in solvents that are less aggressive than NMP, we included the FLG directly onto the surface of already prepared, commercial polymeric films. This facile swellingdeposition approach is interesting industrially because, as it is a post-processing step, it does not require modification of the production setup, and ensures a more uniform distribution of graphene in the first layers of the matrix. First, we repeated the experiments described in Ref. [31], soaking natural rubber in G/PDI dispersions. The rubber was soaked for $12 \mathrm{~h}$ at room temperature in the GPDI-F dispersion in chloroform, and dried for another $12 \mathrm{~h}$ in the open air. After this process, the rubber showed measurable electrical resistance that changed with rubber stretching, going from 300 kW to $3.7 \mathrm{MW}$ upon 300 \% elongation. Similar experiments per formed using G-PDI in THF, instead led to complete dissolution of the natural rubber.

We then used the same approach on commercial films of poly(vinyl chloride) (PVC); this is one of the most technologically relevant polymers, used extensively for pipes, cables, building, clothing, and so on. Transparent sheets of PVC (thickness $182 \pm 4 \mathrm{~mm}$ ) were dipped into chloroform dispersions of G-PDI@F at room temperature, then dried in air to let the chloroform evaporate. The swelling process was completed in less than $3 \mathrm{~min}$, and gave an increase in volume of around

$80 \%$. No further change to the swollen area/size was observed upon leaving the polymer films in the dispersions for more than $3 \mathrm{~min}$. After the swelling treatment, the PVC samples showed a dark color attributed to the presence of the G-PDI@F (Figure 6 b), as well as a bright fluorescence caused by the PDI (Figure $6 \mathrm{c}$ ).

Similar results were obtained with G-PDI@H and G-PDI-Cl in CHCl3 dispersions. Optical absorption spectra of the G-PDI@ PVC multilayers showed the spectral signature of both graphene and PDI, similarly to those observed in dispersion (Figure S5, Supporting Information), with no significant shift in ab- sorption.[30b]

The presence of FLG in the polymer was confirmed by cross- section SEM images. Upon swelling in G-PDI@F dispersions in chloroform, the SEM images show a dense coating of rectangular and polygonal shapes covering the surface (Figure $6 \mathrm{~d}$ and S6, Supporting Information). The sheets were not just de- posited on the surface, but embedded in it, in some cases reaching several micrometers in depth $(<10 \mathrm{~mm})$. Control experiments with swelling only in PDI gave a surface covered with PDI crystals, and no improvement in electrical conductivity (Figures S7, S8, Supporting Information).

AFM images recorded on the PVC films (Figure S9, Supporting Information) showed folded and flat sheets visible on the otherwise rough surface. Typically, 2 D sheets end up lying flat on a substrate upon deposition by conventional solution processing.[32] Owing to the swelling, it is instead clearly observable by SEM that the sheets penetrate into the polymer in an isotropic way, in some cases protruding out of the surface (Figure S6, Supporting Information).

A strong improvement in material hydrophobicity $(\approx 40 \%)$ was observed in all cases, with measurements of water contact angle (Figure S10, Supporting Information) increasing from 728 (blank PVC) to 1038 after treatment with PDI-H and PDI-F and 1018 upon treatment with PDI-F. 
The embedded sheets forms a continuous, micrometer-thick layer on the surface of the otherwise insulating PVC. All three coating films showed similar electrical conductivities with small systematic differences. The sheet resistance $\left(R_{\text {sh }}\right)$ was measured on different areas of each sample using a four probe Van der Paw setup; the most conductive coatings on PVC were obtained using G-PDI-H (i.e. lowest $R_{\text {sh }}=3 \pm 1 \vee 10^{5} \mathrm{~W} / \mathrm{sq}$ ), whereas G-PDI@F gave values of $4.1 \pm 0.9 \vee 105 \mathrm{~W} / \mathrm{sq}$ and G- PDI-Cl gave values of $4.8 \pm 0.9 \mathrm{~V} 105 \mathrm{~W} / \mathrm{sq}$. Such values render these G-PDI materials suitable as polymer additives for antistatic coatings.

\section{CONCLUSION}

All the results obtained to date for LPE of graphite into graphene relied on the use of organic solvents with high surface tension in the range $40-50 \mathrm{~mJ} \mathrm{~m}^{-2}$.[9] However, solvents featuring such high surface tensions also possess high boiling points, and have some limitations in the use of graphene (e.g. , toxicity, or compatibility with industrial standards).

In this study, we have demonstrated that it is possible to combine the use of organic solvents and PDI organic surfactants to obtain dispersions of FLG in solvents in which graphene would not be stable on its own. The results described here provide evidence that few-layer graphene flakes can be stabilized in organic solvents by adding small amounts of suit- able molecules, in a similar but different way to the typical action of soaps in water. The composite materials thus obtained can be included in a straightforward way in elastomer or thermoplastic materials, rendering them conductive. We underline that the process could be industrially relevant because the employed surfactants are commercial PDI molecules that are already used as polymer additives. The process described here could be compatible with large-scale industrial application in the polymer industry. $[13,25]$

Exfoliation in liquids using solvents is one of the most promising techniques for the mass production of graphene and its processing into useful materials. Although graphene produced in this way is already commercially available on an industrial scale,[6] its penetration in the market of advanced materials is still shallow.[33] The main challenges to be overcome are the quality and reliability of the materials produced (larger sheet size, lower average thickness, lower defect density, etc.) and the development of procedures to process graphene in a way that is compatible with actual production standards (com- pounding with polymers, coating on metals and surfaces, integration with silicon-based electronics, etc.). Expanding the range of solvents that can be used for graphene processing could help progress in this direction.

\section{EXPERIMENTAL SECTION}

\section{Materials}

Graphite powder and PDI were purchased from Sigma Aldrich and used as received. The dye molecules used were : 1,3,8,10(2 H,9 H)- tetraone, 2,9-bis(2-phenylethyl)anthra[2,1,9-def :6,5,10d'e'f']diisoquinoline, 2,9-bis[2-(4-fluorophenyl)ethyl]anthra[2,1,9-def :6,5,10- d'e'f']diisoquinoline1,3,8,10(2 $\mathrm{H}, 9 \mathrm{H})$ tetrone, and 2,9-bis[2-(4-chlorophenyl)ethyl]anthra[2,1,9-def $\quad: 6,5,10$ $\mathrm{d}^{\prime} \mathrm{e}^{\prime} \mathrm{f}^{\prime}$ ]diisoquinoline 1,3,8,10(2 H,9 H)tetrone. For simplicity, the molecules are named PDI-H, PDI-F, and PDI-Cl, respectively, in which PDI stands for the perylene diimide core, and the letter corresponds to the final end atom of the phenylethyl side groups (Figure 1). 


\section{Dye capture experiments}

PDI solutions with fixed concentrations were exposed to increasing amounts of graphite flakes with surface area of $6.5 \mathrm{~m}^{2} \mathrm{~g}^{-1}$ (as measured from the producer, Aldrich graphite nanopowder, particle size below $20 \mathrm{~mm}$ ). The dye solutions were exposed to graphite powder for five days, with gentle stirring to prevent artefacts originating from diffusion limits.

\section{Exfoliation experiments}

Mixtures of graphite $\left(3 \mathrm{mg} \mathrm{mL}^{-1}\right)$ and PDI $\left(0.1 \mathrm{mg} \mathrm{mL}^{-1}\right)$ were added to the selected solvent. The solutions were sonicated for $4.5 \mathrm{~h}$ (Elmasonic $\mathrm{P} 70 \mathrm{H}$, frequency $=37 \mathrm{KHz}$, power $=110 \mathrm{~W}$ ) at a constant temperature of $50 \mathrm{8C}$. After sonication, the macroscopic, non-exfoliated graphite particles were eliminated from the dispersions by mild centrifugation at $2200 \mathrm{rpm}$ for $45 \mathrm{~min}$ (Omnifuge 2 RS).

\section{Absorption measurements}

Absorption data were recorded with an Agilent Cary 7000 Universal Measurement Spectrophotometer (UMS) in the wavelength range 300-800 $\mathrm{nm}$. The studied dispersions were then spin-coated on native silicon oxide (SiOx) substrates using a Laurell WS-650MZ-23NPB spin coater. Finally, the substrates were sonicated at $608 \mathrm{C}$ for $15 \mathrm{~min}$ in Acetone and IPA, followed by treatment in a Diener Plasma Cleaner to remove any remaining organic material on the SiOx surface.

\section{STM measurements}

The substrates were put on a magnetic disk by using silver paint glue (Aldrich Chemicals) for both the mechanical and electrical contacts. PDI molecules were dissolved in $\mathrm{CHCl} 3$ and then diluted in 1phenyloctane $(0.08 \mathrm{~mm})$. A drop of solution was cast on the sample just before the measurement. The STM tips were cut mechanically from a Pt/Ir wire $(90 / 10$, diameter $0.25 \mathrm{~mm}$, commercially available from Goodfellow.uk). The raw STM data were processed through the application of background flattening (2nd degree least-square polynomial fitting on $X$ and $Y$ directions), and the drift was corrected by using the underlying graphite lattice as a reference. The latter lattice was visualized by lowering the bias voltage to $20 \mathrm{mV}$ and raising the current to $65 \mathrm{pA}$.[34]

\section{AFM topography}

AFM measurements were performed with a Digital Instruments AFM (Aura NT-MDT), using cantilevers in semicontact mode (tap- ping mode, model RTESP, material : 1-10 W cm phosphorus (n)- doped Si, f0 : 27-309 kHz, k : 20-80 N m${ }^{-1}$; from Bruker). Raw AFM data were treated to remove experimental artifacts by using histogram flattening procedures.[35]

\section{SEM}

SEM images were acquired with a ZEISS 1530 instrument. STM studies of the self-assembly in two dimensions were performed using a Veeco scanning tunneling microscope (multimode Nanoscope III, Veeco) at the interface between highly oriented pyrolitic graphite (HOPG) and a supernatant solution. Dispersions of the investigated molecules were deposited on the basal plane of the surface.

The static water contact angles (CA) were measured at $258 \mathrm{C}$ in air by using a contact angle meter (GBX Digidrop instrument) through the sessile drop method. Each experimental point reported in the graphs was determined as the average value of at least three measurements for each point. The volume of the deionized (DI) water used for these measurements was $1 \mathrm{~mL}$. 
The sheet resistance of the film was measured with a four-point probe system in Van Der Pauw geometry (probed area : $10 \mathrm{~V} 10 \mathrm{~mm}, \mathrm{H} 50$ Hall Effect Controller, MMR technologies).

\section{ACKNOWLEDGEMENTS}

We thank Dr. A. Ciesielski for help with STM imaging. This study was supported by the European Commission through the EC Marie-Curie ITN-GENIUS (PITN-GA-2010-264694), the Flagship GrapheneCore1 (GA-696656), the ANR through the LabEx CSC (ANR-10-LABX-0026 CSC), and the International Center for Frontier Research in Chemistry (icFRC)

KEYWORDS: adsorption, exfoliation, graphene, graphene-based composites, surfactants 


\section{TABLES}

Table 1. Amount of PDI and FLG in THF, estimated by OS.

\begin{tabular}{|c|c|c|c|}
\hline $\begin{array}{l}\text { PDI } \\
\text { Molecule }\end{array}$ & $\begin{array}{l}\text { PDI conc. } \\
\left.\text { [mg L }{ }^{@ 1}\right]\end{array}$ & $\begin{array}{l}\text { FLG conc. } \\
{\left[\mathrm{mgL}^{@ 1}\right]}\end{array}$ & $\begin{array}{l}\mathrm{R}^{[\mathrm{a}]} \\
(\text { from OS) }\end{array}$ \\
\hline PDI@H & 70.78 & 1.58 & 1 \\
\hline PDI@F & 74.00 & 31.63 & 22 \\
\hline PDI@CI & 39.31 & 15.87 & 22 \\
\hline
\end{tabular}

[a] $\mathrm{C}$ atoms of graphene/PDI molecules.

Table 2. Amount of PDI and $\mathrm{FLG}$ in $\mathrm{CHCl}_{3}$ solution, estimated by $\mathrm{OS}$ and STM measurements.

\begin{tabular}{lllll}
$\begin{array}{l}\text { PDI } \\
\text { Molecule }\end{array}$ & $\begin{array}{l}\text { PDI conc. } \\
{\left[\mathrm{mg} \mathrm{L}^{@ 1} \text { ] }\right.}\end{array}$ & $\begin{array}{l}\text { FLG conc. } \\
{\left[\mathrm{mgL}^{@ 1} \text { ] }\right.}\end{array}$ & $\begin{array}{l}\mathrm{R}^{[\mathrm{a}]} \\
\text { (from OS) }\end{array}$ & $\begin{array}{l}\mathrm{R}^{[\mathrm{b}]} \\
(\text { from STM) }\end{array}$ \\
\hline PDI@H & 12.36 & 15.50 & 62 & 37 \\
PDI@F & 11.14 & 11.71 & 55 & 38 \\
PDI@Cl & 9.42 & 11.55 & 68 & 32
\end{tabular}

[a] $R=$ carbon atoms of graphene present in solution/PDI molecule. [b] Assuming perfect PDI coating and perfect exfoliation, leading to $100 \%$ monolayers. 
FIGURES

a) PDI-X

$(\mathrm{X}=\mathrm{H}, \mathrm{F}, \mathrm{Cl})$
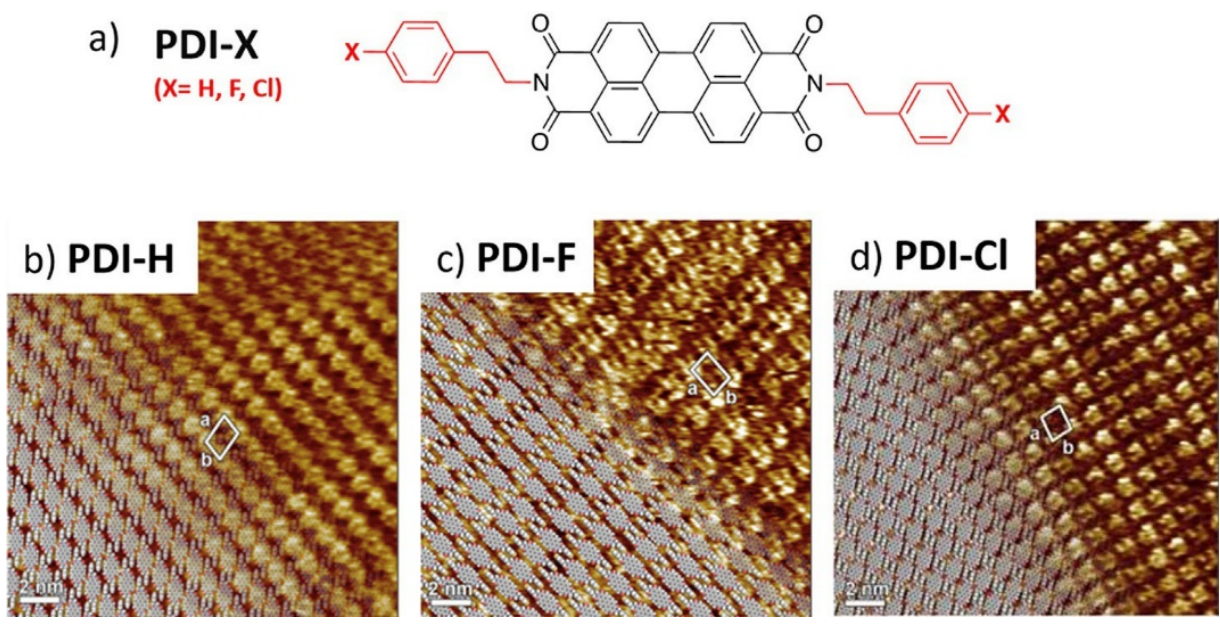

Figure 1. a) Chemical structure of PDI@X, with side chains symmetrically terminated with a different atom (i.e. , H, F, and $\mathrm{Cl}$ ). b-d) STM images of the corresponding 2D supramolecular architectures physisorbed on HOPG at the solid/liquid interface. Scale bar $=2 \mathrm{~nm}$.
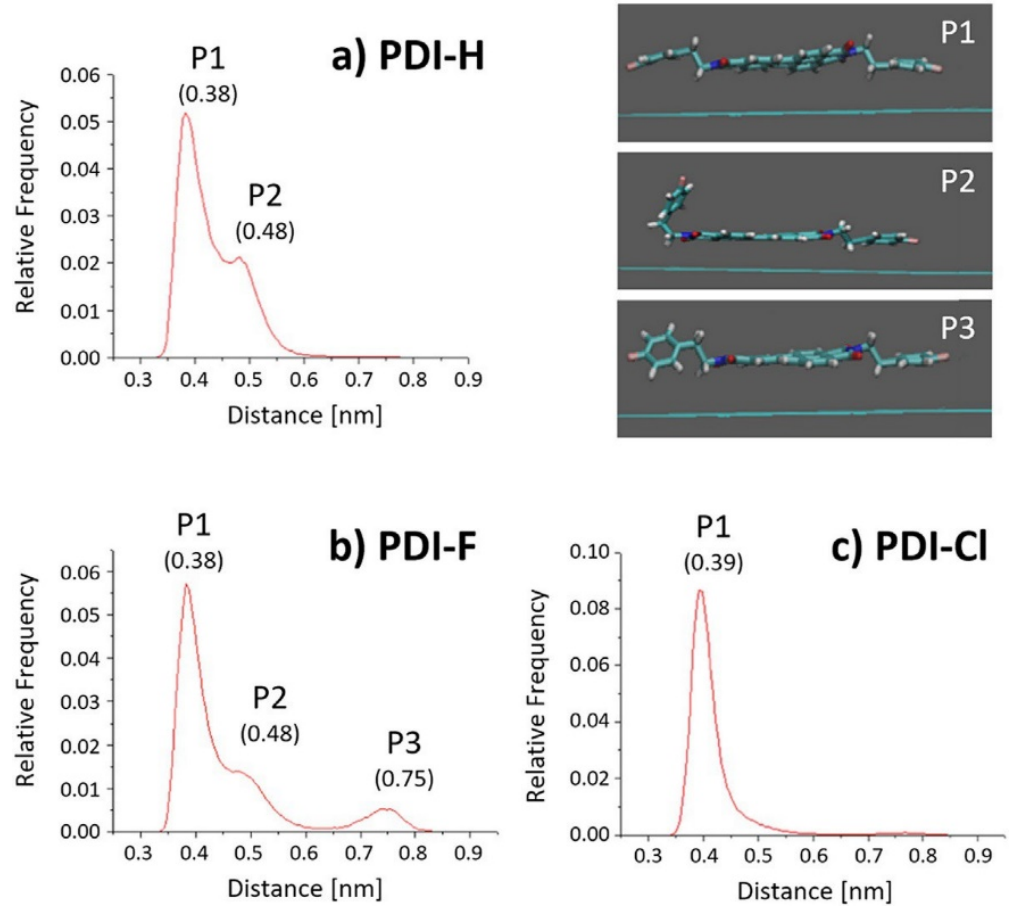

Figure 2. Population distribution of conformers for PDI@F on graphene in chloroform : P1) parallel to graphene, P2) phenyl rings rotate perpendicular to the graphene surface, and P3) phenyl ring folds onto the PDI core. $a, b, c)$ Histogram distribution of the average distance from graphene of the side groups (peak position shown in parentheses) measured for each PDI molecule. 

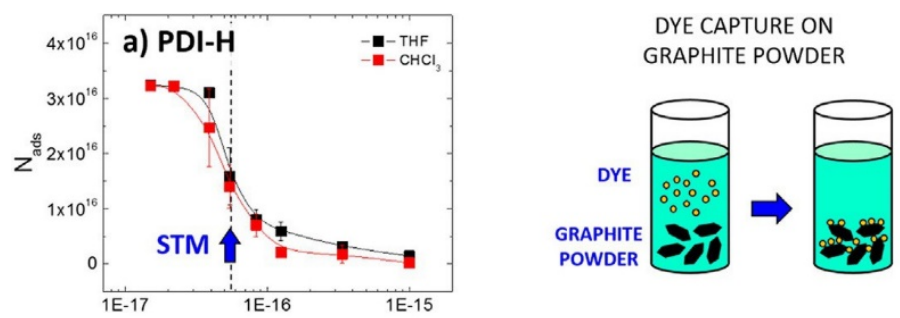

$1 /$ Surface Area $\left[1 / \mathrm{nm}^{2}\right]$
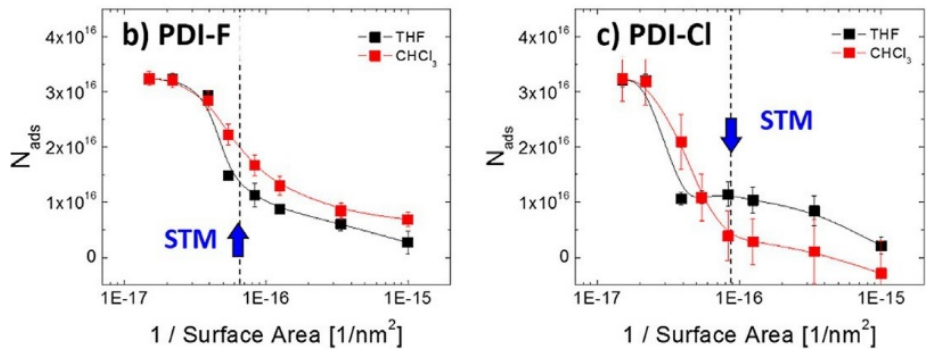

Figure 3. a-c) Number of molecules adsorbed upon varying the available graphite sur-face for the three molecules studied, in THF and chloroform. Initial number of molecules available for adsorption : $3.25 \mathrm{~V} 10^{16}$. The values of surface density calculated by STM are reported for comparison. Black and red lines are a guide for the eye. Cartoon shows a representation of "dye capture" from solution to the graphite substrate.
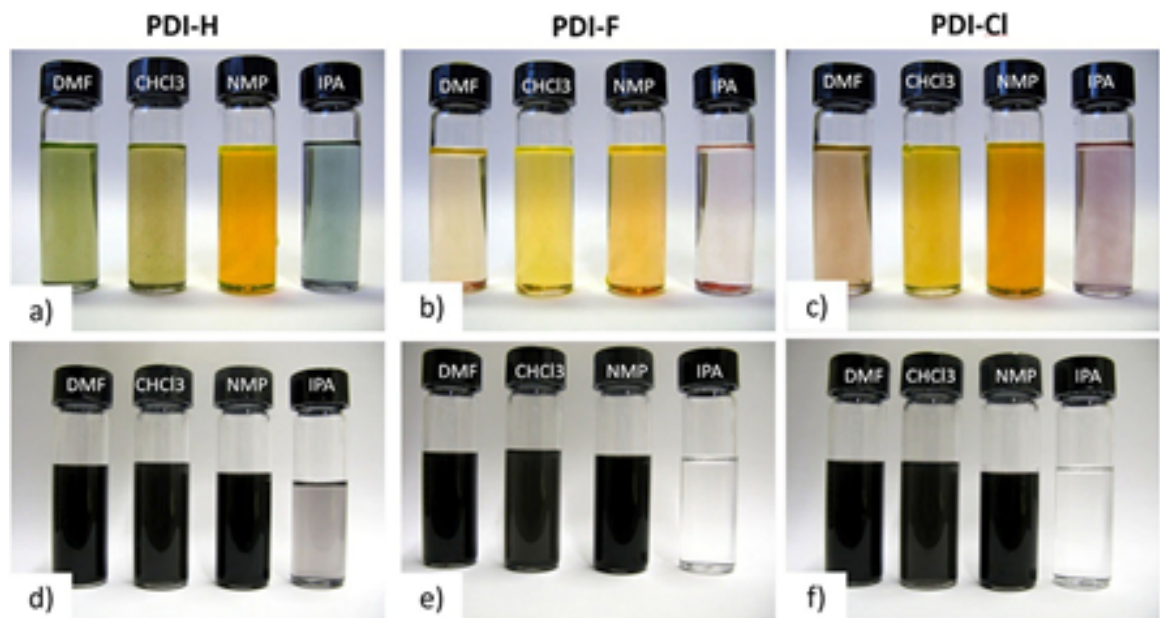

Figure 4. Dispersions of a) PDI-H, b) PDI-F, and c) PDI-Cl in different organic solvents. $d-f$ ) The same solutions after addition of graphite powder, sonication, and centrifugation. 


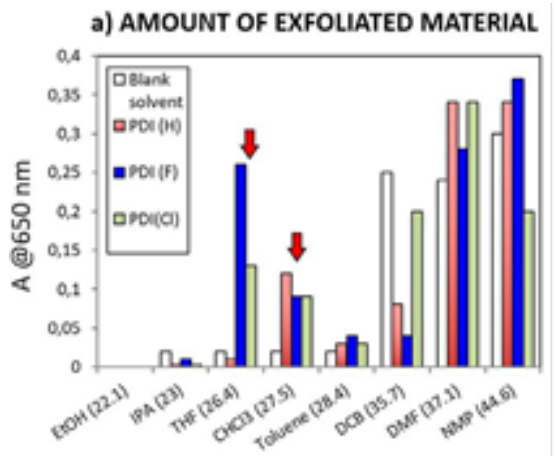

b) RELATIVE INCREASE IN EXFOLIATION

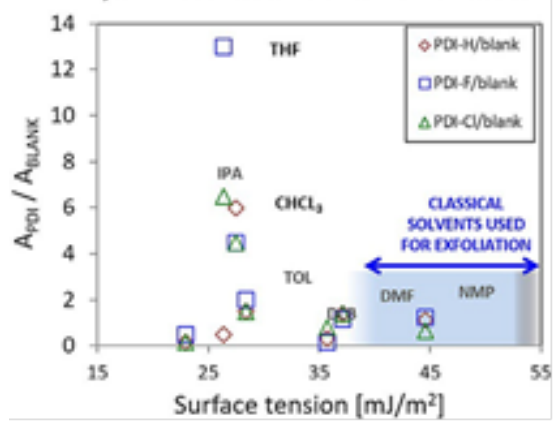

Figure 5. Plots of G-PDIs exfoliated in different solvents related to a) mass of exfoliated material and b) relative increase compared with exfoliation in pure solvent. The shadowed area indicates the surface tension of solvents typically used for graphene exfoliation.

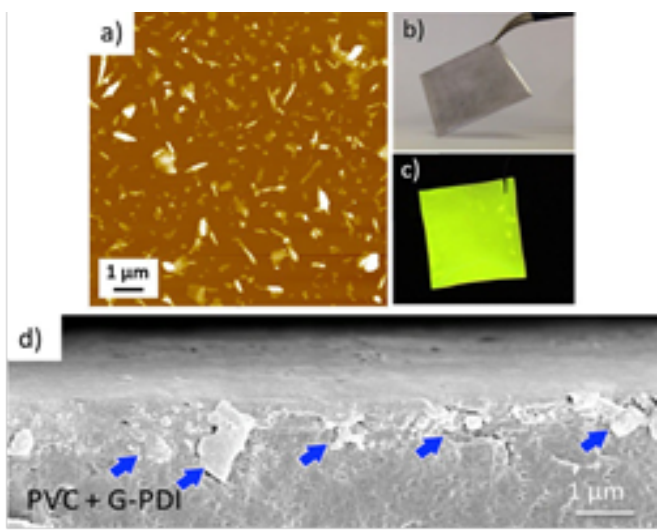

Figure 6. a) Representative image of nanosheets exfoliated with PDI observed by AFM on a SiOx surface. b) Representative image of a PVC film after the swelling process in G-PDI@F solution in $\mathrm{CHCl} 3$. c) The same PVC film under UV light, revealing the fluorescence attributed to PDI molecules. d) Cross-section SEM image of the composite, confirming the presence of intercalated nanosheets embedded in the first micrometer of the surface. 


\section{BIBLIOGRAPHY}

[1] D. Malko, C. Neiss, F. ViÇes, A. Gçrling, Phys. Rev. Lett. 2012, 108, 086804.

[2] S. H. Zhang, J. Zhou, Q. Wang, X. S. Chen, Y. Kawazoe, P. Jena, Proc. Natl.Acad. Sci. USA 2015, 112, 23722377.

[3] R. J. Young, M. Liu, J. Mater. Sci. 2016, 51, $3861-3867$.

[4] a) V. Palermo, Chem. Commun. 2013, 49, 2848 -2857; b) R. K. Joshi, S. Al- warappan, M. Yoshimura, V. Sahajwalla, Y. Nishina, Appl. Mater. Today 2015, 1, 1-12.

[5] K. R. Paton, E. Varrla, C. Backes, R. J. Smith, U. Khan, A. O’Neill, C. Boland, M. Lotya, O. M. Istrate, P. King, T. Higgins, S. Barwich, P. May, P. Puczkarski, I. Ahmed, M. Moebius, H. Pettersson, E. Long, J. Coelho, S. E. O’Brien, E. K. McGuire, B. M. Sanchez, G. S. Duesberg, N. McEvoy, T. J. Pennycook, C. Downing, A. Crossley, V. Nicolosi, J. N. Coleman, Nat. Mater. 2014, 13, $624-630$.

[6] V. Palermo, I. A. Kinloch, S. Ligi, N. M. Pugno, Adv. Mater. 2016, 28, $6232-6238$.

[7] C. G. Liu, Z. N. Yu, D. Neff, A. Zhamu, B. Z. Jang, Nano Letters. 2010, 49, 4863-4868.

[8] Z. Y. Xia, S. Pezzini, E. Treossi, G. Giambastiani, F. Corticelli, V. Morandi, A. Zanelli, V. Bellani, V. Palermo, Adv. Funct. Mater. 2013, 23, 4684 -4693. [9] Y. Hernandez, V. Nicolosi, M. Lotya, F. M. Blighe, Z. Y. Sun, S. De, I. T. Mc- Govern, B. Holland, M. Byrne, Y. K. Gun'ko, J. J. Boland, P. Niraj, G. Dues- berg, S. Krishnamurthy, R. Goodhue, J. Hutchison, V. Scardaci, A. C. Ferrari, J. N. Coleman, Nat. Nanotechnol. 2008, 3, 563 -568.

[10] a) L. Guardia, M. J. Fernandez-Merino, J. I. Paredes, P. Solis-Fernandez, S. Villar-Rodil, A. Martinez-Alonso, J. M. D. Tascon, Carbon 2011, 49, $1653-1662$; b) M. Lotya, A. Rakovich, J. F. Donegan, J. N. Coleman, Nanotech- nology 2013, 24, 265703.

[11] E. Otyepkov\#, P. Lazar, K. C² 8pe, O. Tomanec, M. Otyepka, Appl. Mater. Today 2016, 5, 142 -149.

[12] a) R. Kabe, X. L. Feng, C. Adachi, K. Mellen, Chem. Asian J. 2014, 9, 3125 -3129 ; b) A. Schlierf, P. Samori, V. Palermo, J. Mater. Chem. C 2014, 2, 3129 -3143.

[13] A. Schlierf, K. Cha, M. G. Schwab, P. Samori, V. Palermo, 2D Mater. 2014, 1, 035006.

[14] H. Yang, Y. Hernandez, A. Schlierf, A. Felten, A. Eckmann, S. Johal, P. Lou- ette, J. J. Pireaux, X. Feng, K. Muellen, V. Palermo, C. Casiraghi, Carbon 2013, 53, 357 -365.

[15] I. McCulloch, M. Heeney, C. Bailey, K. Genevicius, I. Macdonald, M. Shku- nov, D. Sparrowe, S. Tierney, R. Wagner, W. M. Zhang, M. L. Chabinyc, R. J. Kline, M. D. McGehee, M. F. Toney, Nat. Mater. 2006, 5, 328 -333.

[16] M. El Gemayel, S. Haar, F. Liscio, A. Schlierf, G. Melinte, S. Milita, O. Ersen, A. Ciesielski, V. Palermo, P. Samori, Adv. Mater. 2014, 26, $4814-4819$.

[17] a) R. Dabirian, V. Palermo, A. Liscio, E. Schwartz, M. B. J. Otten, C. E. Fin- layson, E. Treossi, R. H. Friend, G. Calestani, K. Mullen, R. J. M. Nolte, A. E. Rowan, P. Samori, J. Am. Chem. Soc. 2009, 131, 7055 -7063 ; b) C. E. Fin- layson, R. H. Friend, M. B. J. Otten, E. Schwartz, J. Cornelissen, R. L. M. Nolte, A. E. Rowan, P. Samori, V. Palermo, A. Liscio, K. Peneva, K. Mellen, S. Trapani, D. Beljonne, Adv. Funct. Mater. 2008, 18, 3947 -3955; c) N. V. Kozhemyakina, J. M. Englert, G. A. Yang, E. Spiecker, C. D. Schmidt, F. Hauke, A. Hirsch, Adv. Mater. 2010, 22, 5483 -5487; d) A. Liscio, G. De Luca, F. Nolde, V. Palermo, K. Mellen, P. Samori, J. Am. Chem. Soc. 2008, 130, 780 ; e) V. Palermo, E. Schwartz, C. E. Finlayson, A. Liscio, M. B. J. Otten, S. Trapani, K. Mellen, D. Beljonne, R. H. Friend, R. J. M. Nolte, A. E. Rowan, P. Samori, Adv. Mater. 2010, 22, E81-E88 ; f) X. W. Zhan, A. Facchetti, S. Barlow, T. J. Marks, M. A. Ratner, M. R. Wasielewski, S. R. Marder, Adv. Mater. 2011, 23, 268 -284.

[18] T. Weil, T. Vosch, J. Hofkens, K. Peneva, K. Mellen, Angew. Chem. Int. Ed. 2010, 49, 9068 -9093 ; Angew. Chem. 2010, 122, $9252-9278$.

[19] a) V. Palermo, P. Samori, Angew. Chem. Int. Ed. 2007, 46, 4428 -4432; Angew. Chem. 2007, 119, 4510 4514 ; b) V. Palermo, M. B. J. Otten, A. Liscio, E. Schwartz, P. A. J. de Witte, M. A. Castriciano, M. M. Wienk, F. Nolde, G. De Luca, J. Cornelissen, R. A. J. Janssen, K. Mellen, A. E. Rowan, R. J. M. Nolte, P. Samori, J. Am. Chem. Soc. 2008, 130, $14605-14614$. 
[20] a) K. S. Mali, J. Adisoejoso, E. Ghijsens, I. De Cat, S. De Feyter, Acc. Chem. Res. 2012, 45, 1309 -1320 ; b) G. De Luca, A. Liscio, F. Nolde, L. M. Sco- laro, V. Palermo, K. Mellen, P. Samori, Soft Matter 2008, 4, 2064 -2070.

[21] a) J. N. Coleman, M. Lotya, A. O’Neill, S. D. Bergin, P. J. King, U. Khan, K. Young, A. Gaucher, S. De, R. J. Smith, I. V. Shvets, S. K. Arora, G. Stanton, H. Y. Kim, K. Lee, G. T. Kim, G. S. Duesberg, T. Hallam, J. J. Boland, J. J. Wang, J. F. Donegan, J. C. Grunlan, G. Moriarty, A. Shmeliov, R. J. Nich olls, J. M. Perkins, E. M. Grieveson, K. Theuwissen, D. W. McComb, P. D. Nellist, V. Nicolosi, Science 2011, 331, $568-571$; b) S. M. Tan, Z. Sofer, J. Luxa, M. Pumera, ACS Catal. 2016, 6, $4594-4607$.

[22] C. Backes, B. M. Szydłowska, A. Harvey, S. Yuan, V. Vega-Mayoral, B. R. Davies, P.-I. Zhao, D. Hanlon, E. J. G. Santos, M. I. Katsnelson, W. J. Blau, C. Gadermaier, J. N. Coleman, ACS Nano 2016, 10, 1589 -1601.

[23] R. J. Smith, P. J. King, C. Wirtz, G. S. Duesberg, J. N. Coleman, Chem. Phys. Lett. 2012, 531, 169 -172.

[24] L. Gong, R. J. Young, I. A. Kinloch, I. Riaz, R. Jalil, K. S. Novoselov, ACS Nano 2012, 6, 2086 -2095.

[25] A. Schlierf, H. F. Yang, E. Gebremedhn, E. Treossi, L. Ortolani, L. P. Chen, A. Minoia, V. Morandi, P. Samori, C. Casiraghi, D. Beljonne, V. Palermo, Nanoscale 2013, 5, $4205-4216$.

[26] S. M. Kozlov, F. ViÇes, A. Gçrling, Carbon 2012, 50, 2482 -2492.

[27] S. M. Kozlov, F. Vines, A. Goerling, Adv. Mater. 2011, 23, $2638-2643$.

[28] T. H. Liu, Y. H. Li, Q. J. Du, J. K. Sun, Y. Q. Jiao, G. M. Yang, Z. H. Wang, Y. Z. Xia, W. Zhang, K. L. Wang, H. W. Zhu, D. H. Wu, Colloids Surf. B 2012, 90, 197-203.

[29] D. Gçrl, X. Zhang, F. Werthner, Angew. Chem. Int. Ed. 2012, 51, 6328 - 6348 ; Angew. Chem. 2012, 124, $6434-6455$.

[30] a) K. Balakrishnan, A. Datar, T. Naddo, J. Huang, R. Oitker, M. Yen, J. Zhao, L. Zang, J. Am. Chem. Soc. 2006, 128, $7390-7398$; b) G. De Luca, A. Liscio, M. Melucci, T. Schnitzler, W. Pisula, C. G. Clark, L. M. Scolaro, V. Palermo, K. Mellen, P. Samori, J. Mater. Chem. 2010, 20, 71- 82.

[31] C. S. Boland, U. Khan, C. Backes, A. O’Neill, J. McCauley, S. Duane, R. Shanker, Y. Liu, I. Jurewicz, A. B. Dalton, J. N. Coleman, ACS Nano 2014, 8, $8819-8830$.

[32] a) K. Kouroupis-Agalou, A. Liscio, E. Treossi, L. Ortolani, V. Morandi, N. M. Pugno, V. Palermo, Nanoscale 2014, 6, 5926 -5933 ; b) A. Liscio, G. P. Ver- onese, E. Treossi, F. Suriano, F. Rossella, V. Bellani, R. Rizzoli, P. Samori, V. Palermo, J. Mater. Chem. 2011, 21, 2924 -2931; c) E. Treossi, M. Melucci, A. Liscio, M. Gazzano, P. Samori, V. Palermo, J. Am. Chem. Soc. 2009, 131, 15576.

[33] M. Peplow, Nature 2015, 522, $268-269$.

[34] M. Tarini, P. Cignoni, C. Montani, IEEE Transactions on Visualization and Computer Graphics 2006, 12, $1237-1244$.

[35] A. Liscio, ChemPhysChem 2013, 14, 1283 -1292. 